\title{
Induction kinetics of the Staphylococcus aureus cell wall stress stimulon in response to different cell wall active antibiotics
}

\author{
Vanina Dengler, Patricia Stutzmann Meier, Ronald Heusser, Brigitte Berger-Bächi, Nadine McCallum*
}

\begin{abstract}
Background: Staphylococcus aureus activates a protective cell wall stress stimulon (CWSS) in response to the inhibition of cell wall synthesis or cell envelope damage caused by several structurally and functionally different antibiotics. CWSS induction is coordinated by the VraSR two-component system, which senses an unknown signal triggered by diverse cell wall active agents.

Results: We have constructed a highly sensitive luciferase reporter gene system, using the promoter of sas016 (S. aureus N315), which detects very subtle differences in expression as well as measuring $>4$ log-fold changes in CWSS activity, to compare the concentration dependence of CWSS induction kinetics of antibiotics with different cell envelope targets. We compared the effects of subinhibitory up to suprainhibitory concentrations of fosfomycin, D-cycloserine, tunicamycin, bacitracin, flavomycin, vancomycin, teicoplanin, oxacillin, lysostaphin and daptomycin. Induction kinetics were both strongly antibiotic- and concentration-dependent. Most antibiotics triggered an immediate response with induction beginning within 10 min, except for tunicamycin, D-cycloserine and fosfomycin which showed lags of up to one generation before induction commenced. Induction characteristics, such as the rate of CWSS induction once initiated and maximal induction reached, were strongly antibiotic dependent. We observed a clear correlation between the inhibitory effects of specific antibiotic concentrations on growth and corresponding increases in CWSS induction kinetics. Inactivation of VraR increased susceptibility to the antibiotics tested from 2- to 16-fold, with the exceptions of oxacillin and D-cycloserine, where no differences were detected in the methicillin susceptible $S$. aureus strain background analysed. There was no apparent correlation between the induction capacity of the various antibiotics and the relative importance of the CWSS for the corresponding resistance phenotypes.

Conclusion: CWSS induction profiles were unique for each antibiotic. Differences observed in optimal induction conditions for specific antibiotics should be determined and taken into account when designing and interpreting CWSS induction studies.
\end{abstract}

\section{Background}

Staphylococcus aureus is a major cause of both nosocomial and community-acquired infections worldwide. Because staphylococci can adapt rapidly to varying environmental conditions they are quick to develop resistance to virtually all antibiotics and multiple-drug resistance, especially in methicillin-resistant $S$. aureus (MRSA), severely restricts antibiotic therapy options. One of the major targets for antimicrobial agents is the

\footnotetext{
* Correspondence: mccallum@imm.uzh.ch

Institute of Medical Microbiology, University of Zurich, Gloriastr. 32, 8006 Zurich, Switzerland
}

bacterial cell envelope, which is a complex, multimacromolecular structure that undergoes highly ordered cycles of synthesis and hydrolysis, in order to facilitate cell division while maintaining a protective barrier against environmental stresses. There are several different classes of antibiotics that target specific cell envelope structures or enzymatic steps of cell wall synthesis (Figure 1).

Many antibiotic resistance phenotypes in S. aureus are influenced by global regulators that control virulence factors, metabolism and/or stress responses [1]. One of the latter is the VraSR system, which triggers the cell 


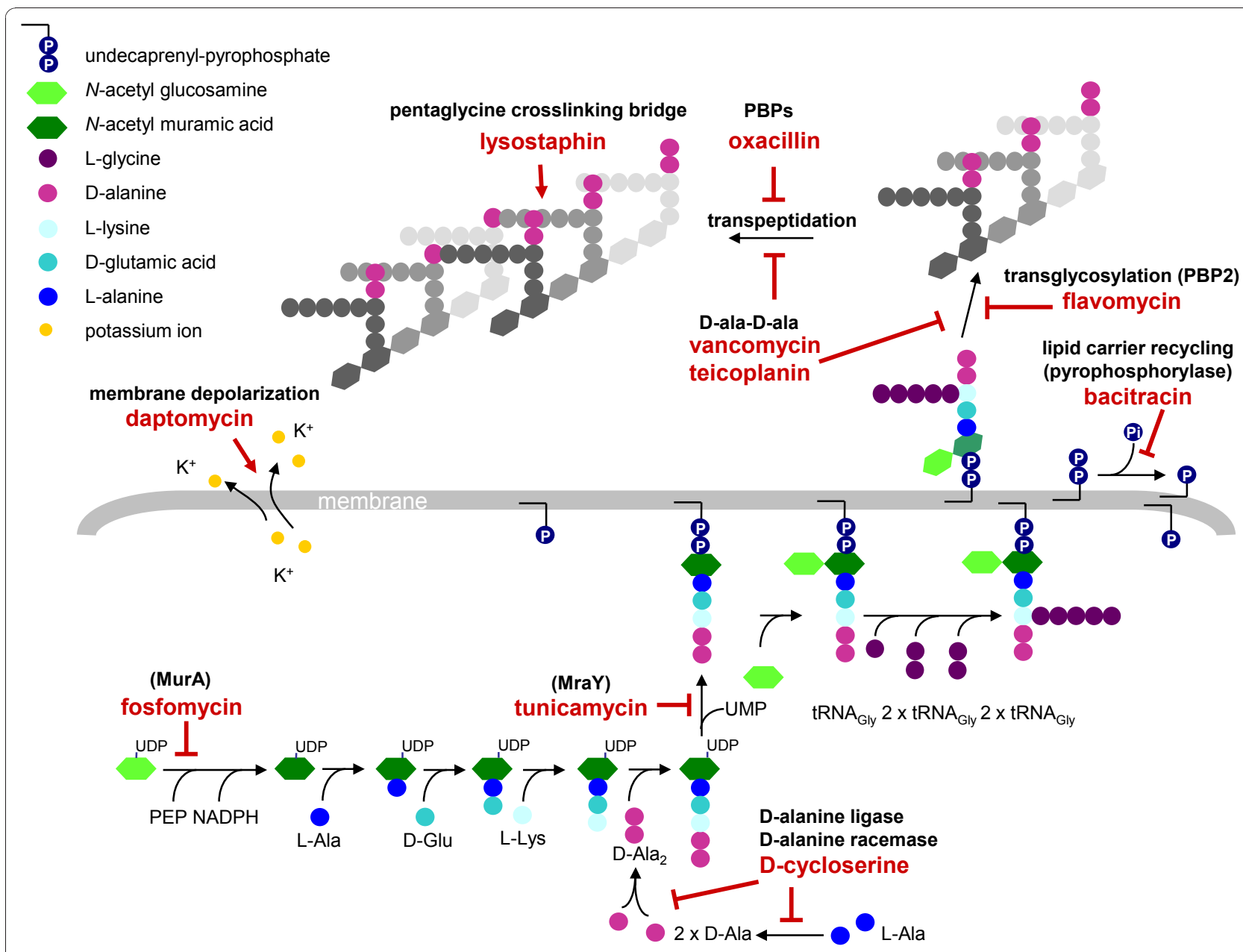

Figure 1 Schematic representation of the enzymatic steps involved in S. aureus cell wall synthesis and the targets of cell wall active antibiotics. Fosfomycin inhibits the enzyme MurA (UDP-N-acetylglucosamine-3-enolpyruvyl transferase) that catalyses the addition of phosphoenolpyruvate (PEP) to UDP-N-acetyl-glucosamine (GICNAc) to form UDP-N-acetyl-muramic acid (UDP-MurNAc) [34]. D-cycloserine prevents the addition of D-alanine to the peptidoglycan precursor by inhibiting D-alanine:D-alanine ligase A and alanine racemase [35]. Tunicamycin is a glycoprotein antibiotic that inhibits the transfer of peptidoglycan precursor (phospho-MurNAc-pentapeptide) to the lipid carrier undecaprenyl pyrophosphate (or C55-isoprenyl pyrophosphate), catalysed by the translocase MraY [36,37]. Sub-lethal concentrations of tunicamycin also inhibit TarO, the first enzyme in the wall teichoic acid pathway [38,39]. Bacitracin forms a metal-dependent complex with the lipid carrier undecaprenyl pyrophosphate, thereby preventing dephosphorylation and the recycling of the lipid carrier required for cell wall synthesis $[40,41]$. Flavomycin (a moenomycin complex) is a phosphoglycolipid antibiotic that inhibits transglycosylation through binding of the transglycosylase domain of penicillin-binding protein 2 (PBP2) [42]. Glycopeptide antibiotics, such as vancomycin and teicoplanin, inhibit cell wall synthesis by binding the D-ala-D-ala of the lipid II and sterically hindering transglycosylation and transpeptidation. Teicoplanin activity is enhanced through its interaction with the cytoplasmic membrane [43]. B-lactam antibiotics, such as oxacillin, bind the transpeptidase active domain of penicillin-binding proteins (PBPs) by mimicking the D-ala-D-ala end of the pentapeptide [44]. The mode of action of daptomycin is not fully known, it causes calcium-dependent disruption of membrane function and potassium efflux [45], but was also predicted to directly or indirectly inhibit peptidoglycan systhesis [9]. Lysostaphin is a zinc metalloenzyme that cleaves the pentaglycine crosslinking bridge specific for the cell wall of S. aureus [46]. (Adapted from [47]).

wall stress stimulon (CWSS); a set of genes that is induced in $S$. aureus upon exposure to cell wall active antibiotics, cell wall hydrolysis, or the inhibition of cell wall synthesis, but not by other external stresses, such as temperature, osmotic or $\mathrm{pH}$ extremes [1-3]. An unknown signal, responding to cell wall stress, stimulates the intramembrane sensor VraS to activate the response regulator VraR by phosphorylation. When the stress signal is relieved, VraR is subsequently deactivated by VraS-specific dephosphorylation [4].

VraR, depending upon its phosphorylation state, was shown to recognise VraR-responsive promoter sequences and to control the expression of target genes [5]. The phosphorylation kinetics suggested that VraSR signal transduction was likely to respond very rapidly in vivo [4]. A general stress signal, rather than the 
antibiotics themselves, was proposed to initiate CWSS induction [6-8]. This hypothesis is supported by the fact that the CWSS is induced by several different cell wall antibiotics with different targets and/or modes of action as well as by the inhibition of cell wall synthesis resulting from reduction of $\mathrm{PBP} 2$ and MurF expression $[6,7,9]$.

Upregulation of the CWSS provides a certain level of resistance/tolerance to most VraSR-inducing agents, although the exact stress response coordinated has not been fully characterised. Core CWSS genes include: murZ (MurA isozyme), involved in the early steps of cell wall biosynthesis [10]; $p b p 2$ and $s g t B$, involved in transglycosylation; and $f m t A$, a penicillin binding protein with low affinity to $\beta$-lactams $[3,11,12]$. Therefore activation of the CWSS is predicted to enhance cell wall synthesis [2]. This is substantiated by the identification of clinical isolates with point mutations in the vraSR operon that lead to increased basal expression of the CWSS in the absence of inducing agents, with the resulting phenotypes including thickened cell walls and increased levels of glycopeptide and $ß$-lactam resistance [13,14].

The VraSR system of $S$. aureus has been found to be induced by a much wider range of cell wall active antibiotics than the homologous LiaRS systems of Bacillus subtilis and Streptococcus mutans, which are only induced by lipid II-interacting antibiotics and not by those that inhibit the earlier or later stages of cell wall synthesis [15-18]. However, the sizes and compositions of VraSR regulons reported so far vary quite extensively and appear to be heavily dependent upon the strains and experimental procedures used. Huge variations in levels of CWSS gene induction were found not only to be dependent upon the types of antibiotic used but also on the antibiotic concentrations $[2,19,20]$.

In this study we created a highly sensitive reporter gene construct to indirectly measure the kinetics of VraSR-dependent signal transduction in the presence of antibiotic concentrations ranging from sub- to supraminimum inhibitory concentrations (MIC), for a selection of antibiotics with different cell envelope targets (Figure 1). This allowed us to compare maximal induction capacities and determine optimal conditions, including concentrations and exposure times, for measuring CWSS induction by different antibiotics.

\section{Methods}

\section{Bacterial strains and growth conditions}

The strains and plasmids used in this study are listed in Table 1 . Bacteria were grown at $37^{\circ} \mathrm{C}$ in Luria Bertani (LB) broth (Difco Laboratories), shaking at $180 \mathrm{rpm}$ with a 1:5 culture to air ratio, or on LB agar plates. All optical density (OD) measurements given were taken at OD $600 \mathrm{~nm}$. Media were supplemented with the following antibiotics when appropriate: $10 \mu \mathrm{g} / \mathrm{ml}$ tetracycline (Sigma), $10 \mu \mathrm{g} / \mathrm{ml}$ chloramphenicol (Sigma), $100 \mu \mathrm{g} / \mathrm{ml}$ ampicillin (Sigma) or $200 \mathrm{ng} / \mathrm{ml}$ anhydrotetracycline (Vetranal). Strains were stored at $-80^{\circ} \mathrm{C}$ in skim milk.

\section{Susceptibility tests}

The MICs of antibiotics were determined by Etest (BioMérieux) on LB plates swabbed with an inoculum of 0.5 McFarland and incubated at $37^{\circ} \mathrm{C}$ for $24 \mathrm{~h}$. The MICs of flavomycin, D-cycloserine, tunicamycin and lysostaphin

Table 1 Strains and plasmids

\begin{tabular}{|c|c|c|}
\hline $\begin{array}{l}\text { Strain/ } \\
\text { plasmid }\end{array}$ & Relevant genotype ${ }^{a}$ & $\begin{array}{l}\text { Reference/ } \\
\text { source }\end{array}$ \\
\hline \multicolumn{3}{|l|}{ S. aureus } \\
\hline RN4220 & Restriction-negative derivative of NCTC8325-4 & {$[48]$} \\
\hline BB255 & NCTC8325 derivative, cured of plasmid pl524 & {$[49]$} \\
\hline BB255 $\triangle$ VraR & BB255 containing vraR mutation, truncating VraR after the $2^{\text {nd }}$ amino acid & This study \\
\hline \multicolumn{3}{|l|}{ E. coli } \\
\hline $\mathrm{DH} 5 \alpha$ &  & Invitrogen \\
\hline \multicolumn{3}{|l|}{ Plasmids } \\
\hline pSP-luct & Luciferase fusion plasmid, ori ColE1, bla, luct; $A p^{r}$ & Promega \\
\hline pBUS1 & E. coli - S. aureus shuttle vector, tetL; $T c^{r}$ & {$[31]$} \\
\hline pKOR1 & $\begin{array}{l}\text { E. coli - S. aureus shuttle plasmid, for creating markerless deletions; repF (ts), cat, attP, ccdB, ori ColE1, bla, Pxyl/tetO, } \\
\text { secY570; } \mathrm{Ap}^{r}, \mathrm{Cm}^{r}\end{array}$ & {$[25]$} \\
\hline $\begin{array}{l}\text { pKOR1-VraR:: } \\
\text { stop }\end{array}$ & $\begin{array}{l}\text { pKOR } 1 \text { construct containing mutant vraR insert with Xhol site and two inframe stop codons inserted between the } \\
2^{\text {nd }} \text { and } 3^{\text {rd }} v \text { raR codons. }\end{array}$ & {$[26]$} \\
\hline psas016p-luct & pBUS1 containing the sas016 promoter-luciferase reporter gene fusion & {$[26]$} \\
\hline ptcaA - luct & pBUS1 containing the tcaA promoter-luciferase reporter gene fusion & This study \\
\hline psa0908 -1 luct & pBUS1 containing the sa0908 promoter-luciferase reporter gene fusion & This study \\
\hline
\end{tabular}

${ }^{a}$ Abbreviations: $\mathrm{Tc}^{r}$, tetracycline resistance; $\mathrm{Ap}^{\mathrm{r}}$, ampicillin resistance; $\mathrm{Cm}^{\mathrm{r}}$, chloramphenicol resistance. 
were determined by microdilution in LB broth, essentially as recommended by the Clinical and Laboratory Standards Institute [21].

\section{Northern Blots}

Northern blots were performed as previously described [22]. Overnight cultures were diluted to OD 0.05 in prewarmed LB containing tetracycline and grown to approximately OD 0.5. Cultures were induced with increasing concentrations of oxacillin and a control culture was grown without antibiotic treatment. Samples were taken after $20 \mathrm{~min}$ and $60 \mathrm{~min}$ of induction and total RNA was extracted as described by Cheung et al. [23]. RNA samples $(7 \mu \mathrm{g})$ were separated in a $1.5 \%$ agarose-20 mM guanidine thiocyanate gel in $1 \times$ TBE buffer [24]. Digoxigenin (DIG)-labelled probes were amplified using the PCR DIG Probe synthesis kit (Roche) and primer pairs SAS016.for (TCATACGTTCTATGTCTGAT) and SAS016.rev (GATCTATATCGTCTTGTAAT); and luc+ (GGCAATCAAATCATTCCGGATACTG) and luc- (ATCCAGATCCACAACCTTCGCTTC).

\section{Construction of vraR mutant}

The pKOR1 system developed by Bae et al. [25] was used to inactivate VraR in BB255, by inserting an XhoI site and two stop codons in-frame into the beginning of the $v r a R$ coding sequence, truncating VraR after the $2^{\text {nd }}$ amino acid, as previously described [26].

\section{Luciferase reporter gene fusions}

Promoter regions of sas016 (SACOL0625), tcaA and sa0908 (SACOL1065) were PCR amplified from S. aureus strain COL using primer pairs: sas016.lucF (AATTAGGTACCTGGATCACGGTGCATACAAC) and sas016.lucR (AATTACCATGGCCTATATTACCTCCTTTGC); $t c a A$. lucF (TAATGGTACCAGTATTAGAAGTCATCAATCA) and tcaA.lucR (TAAT CCATGGTTTCACCTCAAT TCTGTTCCT), and sa0908.lucF (AATTAGGTACCA TAA TAGTACACACGCATGT) and sa0908.lucR (TTAATCCATGGTTGATGCTCCTA TATTAAATT), respectively. PCR products were digested with Asp718 and $\mathrm{NcoI}$ and ligated directly upstream of the promoterless luciferase $(l u c+)$ gene in vector pSP-luc+ (Promega). Fragments containing the resulting promoter-luc+ translational fusions were then excised with Asp718 and EcoR1 and cloned into the E. coli - S. aureus shuttle vector pBUS1 (Table 1). The fusion plasmids ptcaA $A_{\mathrm{p}}-l u c+$, psa0908 ${ }_{\mathrm{p}}-l u c+$ and psas016 $\mathrm{p}-l u c+($ Table 1) were then electroporated into $S$. aureus RN4220 before being transduced, by phage $80 \alpha$, into $S$. aureus BB255.

Luciferase assays for quantification of promoter induction For induction assays, pre-warmed LB broth was inoculated with an overnight culture to an OD of 0.05.
Cultures were grown to OD $0.3-0.5$ and pre-induction samples were collected before the cultures were induced with increasing concentrations of the antibiotics: fosfomycin (disodium salt, Sigma), D-cycloserine (Sigma), bacitracin (from Bacillus lincheniformis, Sigma), vancomycin (Vancocin, Eli Lilly), teicoplanin (Hoechst Marion Roussel), oxacillin (InfectoPharm), flavomycin (BC Biochemie $\mathrm{GmbH}$ ), daptomycin (Cubist Pharmaceuticals), tunicamycin (AG Scientifics) and lysostaphin (ambicin, AMBI). Medium was supplemented with $25 \mu \mathrm{g} / \mathrm{ml} \mathrm{ZnCl}$ for bacitracin, $50 \mu \mathrm{g} / \mathrm{ml} \mathrm{CaCl}{ }_{2}$ for daptomycin and $25 \mu \mathrm{g} / \mathrm{ml}$ glucose-6-phosphate for fosfomycin experiments. Samples were then collected and the OD measured after 10, 20, 30, 45, 60 and $120 \mathrm{~min}$. For each sample, $1 \mathrm{ml}$ of culture was harvested by centrifugation and the pellets frozen at $-20^{\circ} \mathrm{C}$. To measure the luciferase activity, pellets were thawed briefly and resuspended in PBS (pH 7.4) to an OD of either 10 or 1 , depending on induction levels. Aliquots of the cell suspensions were then mixed with equal aliquots of Luciferase Assay System substrate (Promega) and luminescence was measured for $15 \mathrm{~s}$ after a delay of 3 s on a Turner Designs TD-20/20 luminometer (Promega) in relative light units (RLU).

For the determination of colony forming units per millilitre $(\mathrm{CFU} / \mathrm{ml}), 1 \mathrm{ml}$ samples of cultures that had been induced for 120 min with 1 xMIC of each antibiotic were harvested by centrifugation. Cell pellets were resuspended in $0.85 \% \mathrm{NaCl}$ and immediately diluted and plated on sheep-blood agar plates.

\section{Results and Discussion}

\section{Comparison of CWSS reporter constructs}

To quantify CWSS induction and follow its time course upon antibiotic exposure, the promoters of the three representative CWSS genes sas016, sa0908 and tcaA, were fused to the luciferase reporter gene and the resulting plasmids were introduced into antibiotic susceptible strain BB255. sas016 encodes a hypothetical protein of unknown function and was the open reading frame (ORF) found to be most strongly up-regulated by cell wall antibiotics in several studies $[3,11,20]$; tcaA encodes a predicted membrane protein that influences glycopeptide resistance and virulence in a nematode model and belongs to the core $S$. aureus CWSS [11,22,27]; and sa0908 encodes an envelope protein that influences lytic behaviour in S. aureus and is one of a family of three LytR-CpsA-Psr proteins that are all induced by cell wall stress (unpublished results). Increasing concentrations of oxacillin were added to exponentially growing cultures containing reporter plasmids, and the OD and luciferase activities were measured over a two hour period (Figure 2). The three promoters were all induced in a concentration dependent manner, with induction lag times becoming shorter 


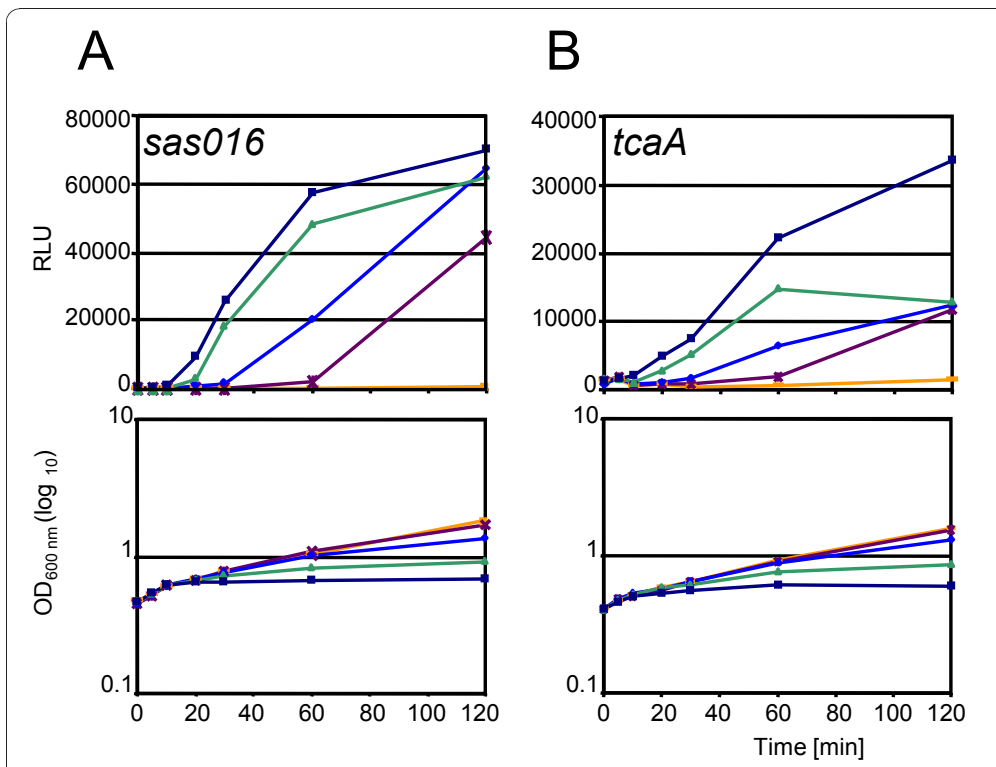

C

Figure 2 Induction kinetics of three CWSS promoters in response to varying concentrations of oxacillin. Luciferase activities and growth curves of strain BB255 containing: A, psas016-luct; B, psa0908-luct; and C, ptcaA-luct; after addition of 0, 0.5, 1, 2 or 5-fold the MIC of oxacillin at time point zero.

and induction rates steeper as oxacillin concentrations increased. This was mirrored by a corresponding stepwise decrease in growth rates. Induction rates generally began to slow after $60 \mathrm{~min}$, upon the onset of oxacillin induced lysis [28], but again this was concentration dependent with induction rates beginning to decrease earlier in cultures with higher oxacillin concentrations.

Previous findings, using Northern blots to measure oxacillin induction levels of sas016 after $30 \mathrm{~min}$, indicated that inhibitory concentrations of oxacillin were required for induction [20]. Figure 2 confirmed that the sub-inhibitory concentration of $0.5 \mathrm{x}$ MIC did not noticeably induce promoter activity after $30 \mathrm{~min}$, however, luciferase activity from all three promoters began to increase sharply after $60 \mathrm{~min}$ and continued to rise up to the final sampling point of $120 \mathrm{~min}$.

Although all three promoters displayed similar relative concentration- and time-dependent induction kinetics, the sas016 promoter produced the highest levels of luciferase activity, resulting in greater fold-changes between samples and making it the most sensitive of the three reporters. Therefore we chose the sas016 promoter-luciferase fusion construct as the best indicator to compare induction characteristics of different cell wall active antibiotics.

\section{Correlation between sas016 transcript induction and luciferase activity from psas016 $\mathrm{p}_{\mathrm{p}}-\mathrm{luc}+$}

To confirm that levels of luciferase activity from psas016 $-l u c+$ accurately represented levels of sas016 gene expression, Northern blots were performed on BB255 psas016 $-l u c+$ RNA samples extracted from cultures grown using the same conditions and oxacillin concentrations used for luciferase assays. Samples were harvested $20 \mathrm{~min}$ and $60 \mathrm{~min}$ after antibiotic induction and hybridized with sas016 and $l u c+$ specific DIG probes (Figure 3). Northern blots showed identical patterns of transcriptional induction for both the chromosomal sas016 gene and the luciferase gene under the control of the sas016 promoter in psas016 $-l u c+$. Induction of both transcripts was highly oxacillin-concentration dependent and transcript intensities increased over time becoming stronger after $60 \mathrm{~min}$ than after $20 \mathrm{~min}$, correlating very well with concentration-specific induction curves from luciferase assays (Figure 2).

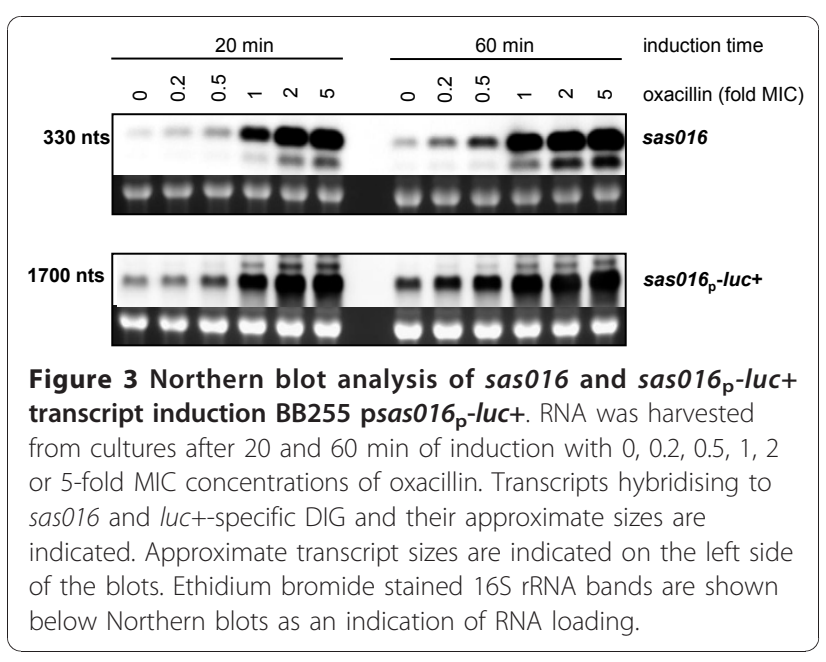




\section{Antibiotic-dependent induction of the CWSS}

The MIC values of diverse antibiotics chosen for induction experiments were determined for strain BB255 psas016 $-l u c+$ (Table 2). MIC concentrations were then used in induction experiments to compare the relative inducing capacities of the antibiotics (Figure 4). When adding MIC concentrations of antibiotics to exponentially growing cultures, salient differences in induction kinetics were apparent throughout the two hour sampling period, including the slopes of induction curves and the maximal luciferase activities reached. Large differences were also seen in the response of the culture's ODs over the induction period, which ranged from slight growth retardation, through to halting of growth and decreasing OD readings; reflecting differences in the effectiveness of the antibiotics and the concentrations used, which are likely to impact CWSS induction kinetics. There were no apparent connections between the stages of cell wall synthesis targeted by antibiotics and CWSS induction potential. Oxacillin and fosfomycin, which target completely different enzymatic stages of peptidoglycan synthesis, showed the highest maximal induction levels, with luciferase activity becoming induced relatively late, but then continually increasing over the two hour period. Bacitracin, tunicamycin, Dcycloserine, flavomycin and teicoplanin showed medium levels of induction, although there were large differences in the shapes of their induction curves. Bacitracin and flavomycin initiated induction very rapidly and maximal expression peaked after $60 \mathrm{~min}$. The teicoplanin induction curve was shallower but maximal induction was again reached at $60 \mathrm{~min}$. Vancomycin was a comparably weak inducer at the MIC concentration. Induction by lysostaphin appeared immediately, within the first $10 \mathrm{~min}$, but remained very low. The OD curve for

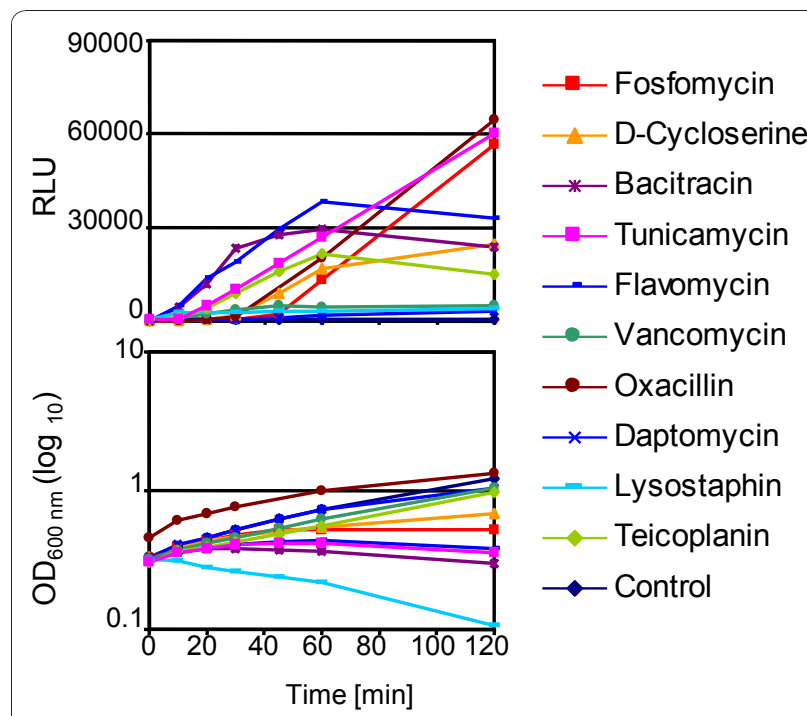

Figure 4 Antibiotic dependent induction of the cell wall stress stimulon. The upper graph shows relative light units (RLU) measured upon induction of BB255 psas016 $-1 u c+$ of cultures stressed with 1X MIC of different antibiotics. The corresponding OD values at each sampling point are presented below. The graphs shown are representative results of between two and four induction experiments performed for each antibiotic.

lysostaphin showed significant lysis of the culture, which would account for the overall low levels of luciferase measured. Induction therefore seems to be more strongly influenced by the specific activities of the different antibiotics used, rather than their targets.

\section{Concentration-dependent CWSS induction kinetics}

Large differences were observed in the CWSS induction kinetics of antibiotics when used at MIC levels, however, these concentrations may not have represented the

Table 2 MIC values and summary of induction kinetics characteristics of different antibiotics

\begin{tabular}{|c|c|c|c|c|c|c|c|}
\hline Antibiotic & $\mathrm{MIC}^{a}$ & $\begin{array}{l}\text { Fold MIC decrease in } \\
\qquad \text { BB255 } \mathrm{VraR}^{b}\end{array}$ & $\begin{array}{l}\text { Lag before } \\
\text { induction }^{c}\end{array}$ & $\begin{array}{l}\text { Maximum } \\
\text { induction }^{d}\end{array}$ & $\begin{array}{c}\text { Time point of } \\
\text { maximum induction }^{e}\end{array}$ & $\begin{array}{l}\text { Concentration } \\
\text { dependence }^{f}\end{array}$ & $\begin{array}{l}\mathrm{OD} / \mathrm{CFU} / \mathrm{ml} \text { as } \% \\
\text { of control }\end{array}$ \\
\hline Fosfomycin & 0.5 & $2 x$ & 30 & high & 120 & high (29.5) & $47 / 10$ \\
\hline $\begin{array}{c}\text { D- } \\
\text { Cycloserine }\end{array}$ & 12 & none & 10 & medium & 60 & high (25.5) & $56 / 36$ \\
\hline Bacitracin & 32 & $10 x$ & none & medium & 60 & low (1.5) & $26 / 9$ \\
\hline Tunicamycin & 8 & $4 x$ & 10 & high & 120 & medium (3.0) & $38 / 9$ \\
\hline Flavomycin & 4 & $16 x$ & none & high & 60 & low (1.6) & $41 / 25$ \\
\hline Vancomycin & 1.3 & $2 x$ & none & low & 120 & medium (12.6) & $100 / 100$ \\
\hline Oxacillin & 0.2 & none & none & high & 120 & high (19.1) & $74 / 20$ \\
\hline Daptomycin & 0.25 & $2 x$ & none & low & 120 & medium (14.1) & $85 / 75$ \\
\hline Lysostaphin & 0.065 & $2 x$ & none & low & 10 & medium (11.3) & $11 / 6$ \\
\hline Teicoplanin & 0.5 & $10 x$ & none & medium & 60 & medium (7.5) & $91 / 83$ \\
\hline
\end{tabular}

${ }^{a}$ Determined in $\mu \mathrm{g} / \mathrm{ml}$ for BB255 psas016-luct. ${ }^{b}$ Difference in MIC values of BB255/BB255 $\Delta$ VraR. ${ }^{c}$ Earliest time point at which induction was detected (min). ${ }^{d}$ Induction levels were scored as: high ( $\left.>40^{\prime} 000 \mathrm{RLU}\right)$; medium ( $\left.>10^{\prime} 000-<40^{\prime} 000\right)$; low $\left(<10^{\prime} 000\right)$. ${ }^{e}$ Time taken for maximum induction to be reached after antibiotic addition (min). ${ }^{f}$ The ratio of maximal induction levels measured at $5 x \mathrm{MIC} / 0.2 \mathrm{x} \mathrm{MIC}$, scored as: high $(>15)$; medium $(>2-<15)$; low $(<2) .{ }^{g}$ OD and $\mathrm{CFU} / \mathrm{ml}$ values after treatment with antibiotics (1x MIC) for $120 \mathrm{~min}$, expressed as a percentage of the values from untreated cell. 
optimal induction conditions for all of the antibiotics. Therefore, induction assays were performed as above, but using five different antibiotic concentrations ranging from sub- up to supra-inhibitory (Figure 5). Additionally, ciprofloxacin, a flouroquinolone antibiotic that does not target the cell envelope was included as a control at concentrations of $2 \mathrm{x}$ and $5 \mathrm{x}$ the MIC $(\mathrm{MIC}=0.2 \mu \mathrm{g} / \mathrm{ml})$.
Tunicamycin, flavomycin, oxacillin and fosfomycin triggered the highest maximal induction levels (RLU > 40’000) (Figure 5A, Table 2). Bacitracin, D-cycloserine, teicoplanin, and vancomycin showed medium levels of induction (RLU > 10'000 - < 40'000), while daptomycin and lysostaphin were the weakest inducers (RLU < 10'000) (Figure 5, Table 2). Daptomycin is known to
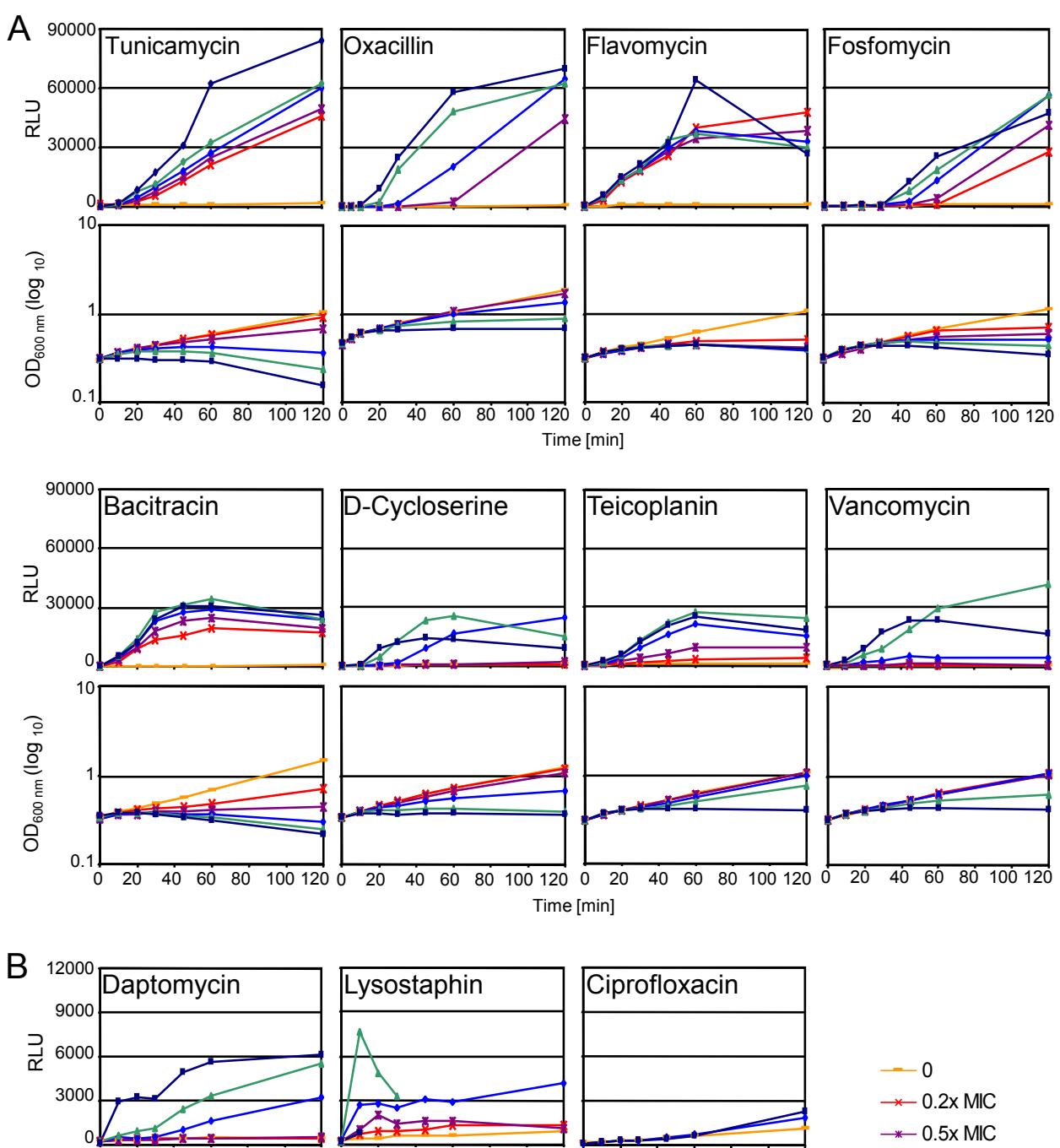

$$
\begin{aligned}
& -0 \\
& -* 0.2 \times \mathrm{MIC} \\
& * 0.5 \times \mathrm{MIC} \\
& \rightarrow 1 \times \mathrm{MIC} \\
& -2 \times \mathrm{MIC} \\
& -5 \times \mathrm{MIC}
\end{aligned}
$$
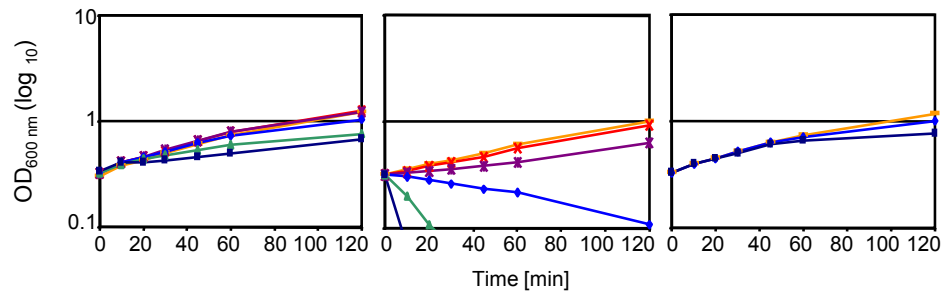

Figure 5 Concentration-dependent cell wall stress stimulon induction kinetics of different cell wall active antibiotics. Graphs show relative light units (RLU) measured upon induction of BB255 psas016p-luc+ with five different antibiotic concentrations and the corresponding OD values at each sampling point. The graphs shown are representative results of between two and four induction experiments performed for each antibiotic. A, concentration-dependent induction kinetics of antibiotics scored as high- or medium-level inducers. B, concentrationdependent induction kinetics of antibiotics scored as low-level inducers and the fluoroquinolone antibiotic ciprofloxacin. 
target the bacterial cell membrane, causing membrane depolarization and potassium efflux (Figure 1), however because of its ability to trigger the CWSS it is also thought to directly or indirectly interfere with peptidoglycan synthesis [9], although the mechanism by which this occurs is unknown.

Ciprofloxacin treated cells showed no luciferase induction after $60 \mathrm{~min}$ and although levels were up to 2-fold higher than in untreated cells after two hours, no further increases in expression were detected, even after four hours when the OD started to decrease in response to the ciprofloxacin treatment. Therefore marginal increases were unlikely to be caused by ciprofloxacinspecific induction of the CWSS as even the lowest inducers, lysostaphin and daptomycin, stimulated 18-fold and 14-fold induction, respectively.

Shapes of the induction curves were different for all of the antibiotics tested. Most of the antibiotics triggered immediate induction of the CWSS, with lysostaphin producing the strongest and most rapid response within the first $10 \mathrm{~min}$, followed by flavomycin, bacitracin, daptomycin, vancomycin, teicoplanin and oxacillin. Contrarily, fosfomycin and D-cycloserine showed a lag phase of induction for all concentrations of approximately $30 \mathrm{~min}$ and $10 \mathrm{~min}$, respectively, before any induction could be detected. Tunicamycin also showed a $10 \mathrm{~min}$ lag phase for all concentrations except $5 x$ MIC, for which a slight 3 -fold induction could be measured at the 10 min sampling point. Fosfomycin, D-cycloserine and tunicamycin act on early steps of peptidoglycan synthesis (Figure 1), which could be linked to the lags in CWSS induction. Balibar et al. also detected a lag phase of CWSS induction when S. aureus was treated with the UPP synthesis inhibitor hymeglusin [29].

Concentration-dependence was categorized based on the spread of the induction curves, so that antibiotics with large distances between the curves for different concentrations were scored as being highly concentration-dependent; while those in which the majority of curves clustered closely together were scored as having low dependence. The concentration-dependency of induction was also evaluated by determining the ratio of the induction measured at $5 \mathrm{x}$ MIC over that at $0.2 \mathrm{x}$ MIC (Table 2). Accordingly, fosfomycin, D-cycloserine, oxacillin, tunicamycin, vancomycin, daptomycin and lysostaphin showed relatively high concentration-dependency (ratio $>2$ ). Some of these antibiotics such as fosfomycin, oxacillin and daptomycin had quite evenly spread curves that generally increased incrementally as concentrations became higher. Whereas for vancomycin, there was a gap between the supra-MIC curves which both showed relatively high induction, and all of the sub-MIC curves that exhibited very little induction. In different experiments curves corresponding to the vancomycin
MIC vacillated between showing either mid-level induction or clustering with the sub-MIC curves, indicating that the MIC of vancomycin was very close to the threshold concentration required for CWSS induction. Flavomycin and bacitracin induction curves also increased incrementally as concentrations increased, but the gaps between the curves were much smaller than for most of the other antibiotics (ratio $<2$ ).

Previous studies have reported contradictory results regarding the induction of the CWSS by lysostaphin. Some studies detected no induction of the CWSS by lysostaphin $[19,30]$, while Rossi et al. detected a slight induction of the CWSS gene mrsR upon lysostaphin treatment [31]. Possible reasons for these discrepancies are likely to be linked to experimental variations in the strains, lysostaphin concentrations and induction times used, or the sensitivity of induction detection methods. In this study, lysostaphin induction could only be detected under very specific experimental conditions (Figure 5B).

The influences of antibiotic concentrations on CWSS induction kinetics generally correlated closely with the impacts of the corresponding concentrations on the OD of the cultures (Figure 5). For example, the incremental increases in oxacillin induction curves closely mirrored corresponding decreases in culture OD curves. For flavomycin, all of the concentrations used induced luciferase activity to similar levels and all growth curves were correspondingly inhibited to similar extents. All experiments showed a definite correlation, albeit to different extents, between levels of growth arrest in the cultures and corresponding levels of CWSS induction. This trend is not always proportional, however, as bacitracin and tunicamycin OD curves showed a large degree of spread whereas induction curves were more closely clustered.

To compare how decreases in OD correlated with cell viability, CFU/ml were measured after treatment with $1 \mathrm{x}$ MIC of each antibiotic for two hours. The percentage decrease in $\mathrm{CFU} / \mathrm{ml}$ generally corresponded well with the percentage decrease in OD (Table 2).

\section{Impact of VraR inactivation on resistance to the cell wall antibiotics tested}

Deletion of the vraSR operon is known to decrease resistance levels to most of its inducing antibiotics $[2,6,9,32]$. However, the reported effects on different resistance phenotypes varied greatly, with some MICs unaffected while others were decreased up to 40 -fold; indicating that induction of the CWSS is more essential for protecting $S$. aureus against some antibiotics than others [2,6,32].

To determine if there was a link between levels or kinetics of CWSS induction and the importance of the CWSS for corresponding resistance phenotypes, we determined the MICs of BB255 compared to BB255 $\Delta$ VraR for all of the antibiotics tested above and 
calculated the fold reduction in MIC (Table 2). BB255 $\Delta$ VraR contains a non-polar deletion truncating VraR after the $2^{\text {nd }}$ amino acid, while leaving the autoregulatory operon intact. The impact of VraR inactivation on resistance phenotypes was very similar to those previously published for deletion of vraSR in S. aureus N315 [2]. The majority of MICs decreased in the VraR mutant compared to the parent strain BB255 (Table 2). The largest impact seen was on the flavomycin MIC, which decreased 16-fold. Bacitracin and teicoplanin MICs were also much lower, with both reduced by 10-fold, and were similar to values previously published for vraSR null-mutants [2]. In contrast to Pietiänen et al. [32], who saw no effects on the vancomycin MIC in a vraSR deletion mutant of strain Newman, we observed a 2-fold decrease in vancomycin MIC, similar to that observed by Kuroda et al. in strain N315 [2]. Our results, which showed a weak 2 -fold reduction in fosfomycin MIC and no impact on D-cycloserine resistance, also agreed with those obtained for the N315 vraSR deletion mutant. While previous reports gave conflicting results concerning the effect of VraSR inactivation on daptomycin resistance [9,32], we observed a reproducible 2-fold reduction in MIC upon VraR inactivation, supporting results from Muthaiyan et al. [9].

Inactivation of VraR had no effect on oxacillin resistance in the methicillin susceptible $S$. aureus (MSSA) strain BB255. However, inactivation of vraR in BB270, an MRSA isogenic to BB255 that contains a type I SCCmec, reduced the oxacillin MIC from $>256$ to 64 $\mu \mathrm{g} / \mathrm{ml}$ [26], to similar levels as those reported for other vraSR mutants in MRSA strains [2,6,33]. Loss of VraR also rendered the mutant 2 -fold more susceptible to the action of lysostaphin and 4-fold more susceptible to tunicamycin; phenotypes which have not been previously published for VraSR mutants.

These results confirmed that the ability to induce the cell wall stress stimulon confers varying levels of protection against the effects of cell wall active agents. However, comparison of our MIC results with our induction data revealed no clear links between how quickly, or to which maximal level, the antibiotics are able to induce the CWSS and the impact of a functional VraSR signal transduction response on resistance levels to those antibiotics.

The sas016 promoter-luciferase fusion construct was also analysed in BB255 $\Delta \mathrm{VraR}$. Expression levels of psas016 $-l u c+$ in BB255 V VraR in uninduced samples were $\sim 10$-fold lower than in the wild type BB255. BB255 V VraR psas016 $-l u c+$ was induced with $5 \mathrm{x}$ MIC of fosfomycin, D-cycloserine, tunicamycin, bacitracin, flavomycin, vancomycin, teicoplanin, oxacillin and daptomycin and $1 \mathrm{x}$ MIC of lysostaphin, for $60 \mathrm{~min}$. The luciferase activities ranged from 1.5-fold higher to 10fold lower than those in uninduced cultures, showing that none of the antibiotics used could induce sas016 expression in absence of VraR.

\section{Conclusions}

In this study, we describe the application of a highly sensitive luciferase-reporter gene construct for indirectly measuring CWSS induction kinetics in S. aureus. This system was used to compare induction characteristics of ten different cell wall active antibiotics with diverse enzymatic targets or modes of action. Induction of the CWSS by all ten antibiotics could be precisely quantified and while all ten antibiotics induced the CWSS, induction patterns varied greatly and were highly antibioticspecific. Each antibiotic produced unique induction curves, which differed in lag times before induction, maximal rates of induction and peak induction levels.

Induction kinetics were also strongly antibiotic concentration-dependent, to different extents for each antibiotic, and generally correlated inversely with decreasing OD values, therefore linking induction kinetics to antibiotic activity. However, there were no obvious trends linking antibiotics acting on similar stages of CWSS with specific induction patterns. Therefore, the signal triggered by all of the antibiotics, that is responsible for activating VraS signal transduction, does not appear to be linked to any particular enzymatic target, as CWSS induction was triggered equally strongly by antibiotics targeting early cytoplasmic stages (e.g. fosfomycin) and late extracellular polymerization stages (e.g. oxacillin) of peptidoglycan synthesis. This is a key difference between the VraSR system of $S$. aureus and the homologous LiaRS systems of other Gram-positive bacteria such as $B$. subtilis and S. mutans, which are only activated by lipid-II interacting antibiotics, such as bacitracin, ramoplanin and nisin [15-18]. The increased induction spectrum could account for the larger size of the $S$. aureus CWSS and its protective role against more different classes of antibiotics. Although no direct links between induction properties and the impact of the CWSS on respective resistance phenotypes could be found.

Previous studies have reported large differences in CWSS induction characteristics. However, most studies were performed on different strains and using different experimental conditions. Variations in characteristics observed for the ten antibiotics tested here, indicated that each antibiotic has optimal induction conditions that should be determined before CWSS studies are carried out, including the right antibiotic concentration for the strain used and the optimal sampling time point to measure maximal induction.

\section{Acknowledgements}

This study has been carried out with financial support from the Commission of the European Communities, specifically the Infectious Diseases research 
domain of the Health theme of the 7th Framework Programme, contract number 241446, "The effects of antibiotic administration on the emergence and persistence of antibiotic-resistant bacteria in humans and on the composition of the indigenous microbiotas at various body sites"; and the Swiss National Science Foundation grant 31-117707.

\section{Authors' contributions}

VD carried out most of the experimental work and drafted the manuscript. PS and BB participated in the design and coordination of the study and helped to draft the manuscript. $\mathrm{RH}$ participated in the microbiological studies and helped to draft the manuscript. NM participated in the design and coordination of the study, carried out molecular biological studies and helped to draft the manuscript. All authors read and approved the final manuscript.

Received: 24 September 2010 Accepted: 20 January 2011

Published: 20 January 2011

\section{References}

1. Jordan S, Hutchings MI, Mascher T: Cell envelope stress response in Gram-positive bacteria. FEMS Microbiol Rev 2008, 32(1):107-146.

2. Kuroda M, Kuroda H, Oshima T, Takeuchi F, Mori H, Hiramatsu K: Twocomponent system VraSR positively modulates the regulation of cellwall biosynthesis pathway in Staphylococcus aureus. Mol Microbio/ 2003 49(3):807-821

3. Utaida S, Dunman PM, Macapagal D, Murphy E, Projan SJ, Singh VK, Jayaswal RK, Wilkinson BJ: Genome-wide transcriptional profiling of the response of Staphylococcus aureus to cell-wall-active antibiotics reveals a cell-wall-stress stimulon. Microbiology 2003, 149(Pt 10):2719-2732.

4. Belcheva A, Golemi-Kotra D: A close-up view of the VraSR twocomponent system. A mediator of Staphylococcus aureus response to cell wall damage. J Biol Chem 2008, 283(18):12354-12364.

5. Belcheva A, Verma V, Golemi-Kotra D: DNA-binding activity of the vancomycin resistance associated regulator protein $\mathrm{VraR}$ and the role of phosphorylation in transcriptional regulation of the vraSR operon. Biochemistry 2009, 48(24):5592-5601.

6. Gardete S, Wu SW, Gill S, Tomasz A: Role of VraSR in antibiotic resistance and antibiotic-induced stress response in Staphylococcus aureus. Antimicrob Agents Chemother 2006, 50(10):3424-3434.

7. Sobral RG, Jones AE, Des Etages SG, Dougherty TJ, Peitzsch RM, Gaasterland T, Ludovice AM, de Lencastre H, Tomasz A: Extensive and genome-wide changes in the transcription profile of Staphylococcus aureus induced by modulating the transcription of the cell wall synthesis gene murF. J Bacteriol 2007, 189(6):2376-2391.

8. McCallum N, Berger-Bachi B, Senn MM: Regulation of antibiotic resistance in Staphylococcus aureus. Int J Med Microbio/ 2009, 300(2-3):118-129.

9. Muthaiyan A, Silverman JA, Jayaswal RK, Wilkinson BJ: Transcriptional profiling reveals that daptomycin induces the Staphylococcus aureus cell wall stress stimulon and genes responsive to membrane depolarization. Antimicrob Agents Chemother 2008, 52(3):980-990.

10. Blake $\mathrm{KL}, \mathrm{O}$ 'Neill AJ, Mengin-Lecreulx D, Henderson PJ, Bostock JM, Dunsmore CJ, Simmons KJ, Fishwick CW, Leeds JA, Chopra I: The nature of Staphylococcus aureus MurA and MurZ and approaches for detection of peptidoglycan biosynthesis inhibitors. Mol Microbiol 2009, 72(2):335-343.

11. McAleese F, Wu SW, Sieradzki K, Dunman P, Murphy E, Projan S, Tomasz A Overexpression of genes of the cell wall stimulon in clinical isolates of Staphylococcus aureus exhibiting vancomycin-intermediate- S. aureustype resistance to vancomycin. J Bacteriol 2006, 188(3):1120-1133.

12. Fan X, Liu Y, Smith D, Konermann L, Siu KW, Golemi-Kotra D: Diversity of penicillin-binding proteins. Resistance factor FmtA of Staphylococcus aureus. J Biol Chem 2007, 282(48):35143-35152.

13. Kato $Y$, Suzuki T, Ida T, Maebashi K: Genetic changes associated with glycopeptide resistance in Staphylococcus aureus: predominance of amino acid substitutions in YvqF/VraSR. J Antimicrob Chemother 2010, 65(1):37-45.

14. Cui L, Neoh HM, Shoji M, Hiramatsu K: Contribution of vraSR and graSR point mutations to vancomycin resistance in vancomycin-intermediate Staphylococcus aureus. Antimicrob Agents Chemother 2009, 53(3):1231-1234

15. Eldholm V, Johnsborg O, Straume D, Ohnstad HS, Berg KH, Hermoso JA, Havarstein LS: Pneumococcal CbpD is a murein hydrolase that requires a dual cell envelope binding specificity to kill target cells during fratricide. Mol Microbiol 2010, 76(4):905-917.

16. Jordan S, Junker A, Helmann JD, Mascher T: Regulation of LiaRS dependent gene expression in bacillus subtilis: identification of inhibitor proteins, regulator binding sites, and target genes of a conserved cell envelope stress-sensing two-component system. J Bacteriol 2006, 188(14):5153-5166.

17. Mascher T, Zimmer SL, Smith TA, Helmann JD: Antibiotic-inducible promoter regulated by the cell envelope stress-sensing two-component system LiaRS of Bacillus subtilis. Antimicrob Agents Chemother 2004, 48(8):2888-2896.

18. Suntharalingam $P$, Senadheera MD, Mair RW, Levesque CM, Cvitkovitch DG: The LiaFSR system regulates the cell envelope stress response in Streptococcus mutans. J Bacterio/ 2009, 191(9):2973-2984.

19. Steidl R, Pearson S, Stephenson RE, Ledala N, Sitthisak S, Wilkinson BJ, Jayaswal RK: Staphylococcus aureus cell wall stress stimulon gene-lacZ fusion strains: potential for use in screening for cell wall-active antimicrobials. Antimicrob Agents Chemother 2008, 52(8):2923-2925.

20. McCallum N, Spehar G, Bischoff M, Berger-Bachi B: Strain dependence of the cell wall-damage induced stimulon in Staphylococcus aureus. Biochim Biophys Acta 2006, 1760(10):1475-1481.

21. Institute CaLS: Performace standards for antimicrobial susceptibility testing. Wayna, PA; 2010, M100-S120, vol. 30.

22. McCallum N, Brassinga AK, Sifri CD, Berger-Bachi B: Functional characterization of TcaA: minimal requirement for teicoplanin susceptibility and role in Caenorhabditis elegans virulence. Antimicrob Agents Chemother 2007, 51(11):3836-3843.

23. Cheung $A L$, Eberhardt $K J$, Fischetti VA: A method to isolate RNA from gram-positive bacteria and mycobacteria. Anal Biochem 1994 222(2):511-514.

24. Goda SK, Minton NP: A simple procedure for gel electrophoresis and northern blotting of RNA. Nucleic Acids Res 1995, 23(16):3357-3358.

25. Bae T, Schneewind O: Allelic replacement in Staphylococcus aureus with inducible counter-selection. Plasmid 2006, 55(1):58-63.

26. McCallum N, Stutzmann Meier P, Heusser R, Berger-Bächi B: Mutational analyses of ORFs within the vraSR operon and their roles in the cell wall stress response of Staphylococcus aureus. Antimicrob Agents Chemother 2011.

27. Maki H, McCallum N, Bischoff M, Wada A, Berger-Bachi B: tcaA inactivation increases glycopeptide resistance in Staphylococcus aureus. Antimicrob Agents Chemother 2004, 48(6):1953-1959.

28. Maidhof $H$, Johannsen L, Labischinski $H$, Giesbrecht P: Onset of penicillininduced bacteriolysis in staphylococci is cell cycle dependent. J Bacteriol 1989, 171(4):2252-2257.

29. Balibar CJ, Shen X, McGuire D, Yu D, McKenney D, Tao J: cwrA, a gene that specifically responds to cell wall damage in Staphylococcus aureus. Microbiology 2010, 156(Pt 5):1372-1383.

30. Pechous R, Ledala N, Wilkinson BJ, Jayaswal RK: Regulation of the expression of cell wall stress stimulon member gene msrA1 in methicillin-susceptible or -resistant Staphylococcus aureus. Antimicrob Agents Chemother 2004, 48(8):3057-3063.

31. Rossi J, Bischoff M, Wada A, Berger-Bachi B: MsrR, a putative cell envelopeassociated element involved in Staphylococcus aureus sarA attenuation. Antimicrob Agents Chemother 2003, 47(8):2558-2564.

32. Pietiainen $M$, Francois $P$, Hyyrylainen $H L$, Tangomo M, Sass $V$, Sahl HG, Schrenzel J, Kontinen VP: Transcriptome analysis of the responses of Staphylococcus aureus to antimicrobial peptides and characterization of the roles of vraDE and vraSR in antimicrobial resistance. BMC Genomics 2009, 10:429.

33. Boyle-Vavra S, Yin S, Daum RS: The VraS/NraR two-component regulatory system required for oxacillin resistance in community-acquired methicillin-resistant Staphylococcus aureus. FEMS Microbiol Lett 2006, 262(2):163-171.

34. Kahan FM, Kahan JS, Cassidy PJ, Kropp H: The mechanism of action of fosfomycin (phosphonomycin). Ann N Y Acad Sci 1974, 235(0):364-386.

35. Lambert MP, Neuhaus FC: Mechanism of D-cycloserine action: alanine racemase from Escherichia coli W. J Bacteriol 1972, 110(3):978-987.

36. Heifetz A, Keenan RW, Elbein AD: Mechanism of action of tunicamycin on the UDP-GIcNAc:dolichyl-phosphate Glc-NAc-1-phosphate transferase. Biochemistry 1979, 18(11):2186-2192.

37. Brandish PE, Kimura KI, Inukai M, Southgate R, Lonsdale JT, Bugg TD: Modes of action of tunicamycin, liposidomycin $B$, and mureidomycin $A$ : 
inhibition of phospho-N-acetylmuramyl-pentapeptide translocase from Escherichia coli. Antimicrob Agents Chemother 1996, 40(7):1640-1644.

38. Swoboda JG, Meredith TC, Campbell J, Brown S, Suzuki T, Bollenbach T, Malhowski AJ, Kishony R, Gilmore MS, Walker S: Discovery of a small molecule that blocks wall teichoic acid biosynthesis in Staphylococcus aureus. ACS Chem Biol 2009, 4:875-883.

39. Wyke AW, Ward JB: Biosynthesis of wall polymers in Bacillus subtilis. J Bacteriol 1977, 130(3):1055-1063.

40. Qi ZD, Lin Y, Zhou B, Ren XD, Pang DW, Liu Y: Characterization of the mechanism of the Staphylococcus aureus cell envelope by bacitracin and bacitracin-metal ions. J Membr Biol 2008, 225(1-3):27-37.

41. Stone KJ, Strominger JL: Mechanism of action of bacitracin: complexation with metal ion and C 55 -isoprenyl pyrophosphate. Proc Natl Acad SCi USA 1971, 68(12):3223-3227.

42. Halliday J, McKeveney D, Muldoon C, Rajaratnam P, Meutermans W: Targeting the forgotten transglycosylases. Biochem Pharmacol 2006, 71(7):957-967.

43. Beauregard DA, Williams DH, Gwynn MN, Knowles DJ: Dimerization and membrane anchors in extracellular targeting of vancomycin group antibiotics. Antimicrob Agents Chemother 1995, 39(3):781-785.

44. Ghuysen JM: Serine beta-lactamases and penicillin-binding proteins. Annu Rev Microbiol 1991, 45:37-67.

45. Baltz RH: Daptomycin: mechanisms of action and resistance, and biosynthetic engineering. Curr Opin Chem Biol 2009, 13(2):144-151.

46. Kumar JK: Lysostaphin: an antistaphylococcal agent. Appl Microbio Biotechnol 2008, 80(4):555-561.

47. McCallum N, Berger-Bachi B, Senn MM: Regulation of antibiotic resistance in Staphylococcus aureus. Int J Med Microbiol 2010, 300(2-3):118-129.

48. Kreiswirth BN, Lofdahl S, Betley MJ, O'Reilly M, Schlievert PM, Bergdoll MS, Novick RP: The toxic shock syndrome exotoxin structural gene is not detectably transmitted by a prophage. Nature 1983, 305(5936):709-712.

49. Berger-Bachi B: Insertional inactivation of staphylococcal methicillin resistance by Tn551. J Bacteriol 1983, 154(1):479-487.

doi:10.1186/1471-2180-11-16

Cite this article as: Dengler et al:: Induction kinetics of the

Staphylococcus aureus cell wall stress stimulon in response to different cell wall active antibiotics. BMC Microbiology 2011 11:16.

\section{Submit your next manuscript to BioMed Central and take full advantage of:}

- Convenient online submission

- Thorough peer review

- No space constraints or color figure charges

- Immediate publication on acceptance

- Inclusion in PubMed, CAS, Scopus and Google Scholar

- Research which is freely available for redistribution

Submit your manuscript at www.biomedcentral.com/submit
C Biomed Central 